\title{
A MAGNETOSTATIC MEMS SWITCH FOR DC BRUSHLESS MOTOR COMMUTATION
}

\author{
John A. Wright and Yu-Chong Tai \\ California Institute of Technology \\ Division of Engineering and Applied Science \\ Pasadena, California 91125 \\ Gerald Lilienthal \\ Jet Propulsion Laboratory \\ Pasadena, California 91109
}

\begin{abstract}
A magnetostatic micromachined switch for commutation of DC brushless motors has been developed. Large contact closure forces $(>5 \mathrm{mN})$ are generated on a permalloy plate by strong permanent magnets attached to the rotating motor shaft. Four point measurements of the switch show a contact resistance of less than 35 milliohms. Commutation of a three-phase, four-pole DC brushless motor by three of the MEMS switches has been successfully demonstrated. In hot-switched, resistive load lifetime testing, no failure is seen after greater than 500 million cycles at low currents $(<1 \mathrm{~mA})$ but at much higher currents $(0.45 \mathrm{~A})$, lifetime is reduced to less than 1 million cycles.
\end{abstract}

\section{INTRODUCTION}

DC brushless motors are widely used in applications ranging from spacecraft to the video players. As is prevalent in many industries, demand for miniaturization is driving motors to much smaller sizes and at the same time requiring improved performance. While reduction of motor size is advancing (non-micromachined motors as small as $3-\mathrm{mm}$-diameter are commercially available [1]), miniaturizing the size of commutation electronics is not keeping pace. Fig-

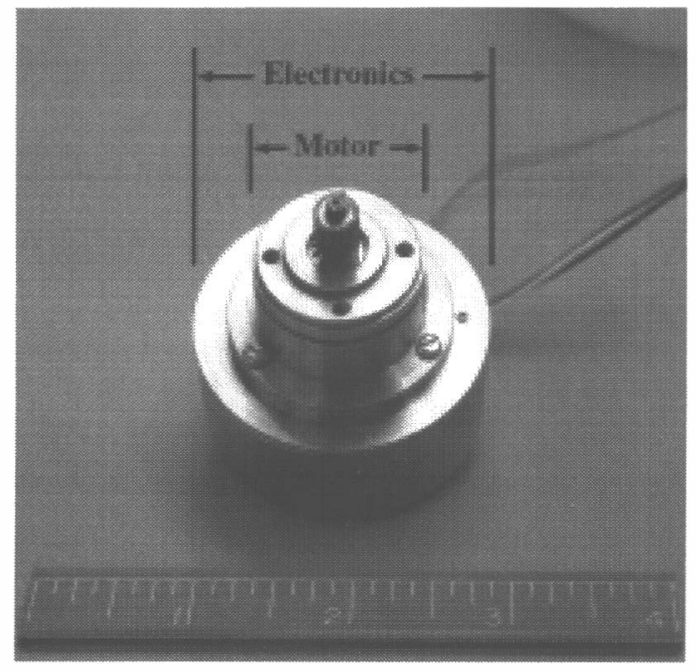

Figure 1. Miniature DC brushless motor mounted to its commutation electronics. The volume of the electronics if four times that of the motor: ure 1 shows a miniature DC brushless motor that is about an inch in diameter. It sits on top of its commutation electronics package which is nearly four times the motor's volume.

Control of DC brushless motors is achieved by a complex package of position sensing and power electronics. To properly operate, a given winding of the motor must be energized only for a portion of the rotor's rotation. If the winding is active otherwise, it retards the motor rotation, decreasing efficiency and increasing torque ripple. For this reason, shaft position must be accurately sensed, usually by either hall sensors or an encoder. The signals from the sensor are used by the commutation electronics to generate the appropriate waveforms for each motor winding. The end result is a complex controller consisting of a hundred or more components occupying a relatively large volume and consuming a notable amount of power. To keep pace with motor miniaturization, reduction of part count and complexity is needed.

Replacement of both position sensor and commutation electronics can be achieved using a single micromachined magnetostatic switch for each motor phase. Utilization of magnetostatic actuation forces has been previously used [2-5] to produce large, out-of-plane deflection devices. Micromachining permits the switches to have nearly negligible size and weight which will allow them to be mounted inside the motor. Mounting the devices inside the motor provides two very attractive benefits. First, the distance of the wires run between the motor and the switches is minimized. Second, and most importantly, actuation and control of the switches can be provided by the motor itself. The magnetic fields produced by the magnets within the motor fluctuates as the motor turns. By proper design and placement of the magnetostatic switches, these fields can act as both the control and the actuating force to open and close the switches while providing perfect synchronization of switching time versus rotor position. By eliminating the need for the sensing and control circuits, system complexity is reduced while reliability is greatly enhanced. No external power source is needed to actuate the switches, saving energy, while reliability is further improved by minimizing the number of wiring connections in the system.

\section{DESIGN}

The proposed micromachined magnetostatic switches, actuated by an external magnetic field consist of three main components: a cantilever-beam spring, a magnetic actuation plate and two electrical contact points. When the switch is inactive, the cantilever beam holds the contact points apart. To activate the switch, an external magnetic field is applied perpendicular to the magnetic actuation plate. Forces are generated that attempt to align the plate with the 
magnetic field, causing the cantilever beam to bend. If the external magnetic field exceeds the design threshold of the switch, the bending of the beam will bring the two electrical contacts together and complete a circuit. The circuit is broken when the external magnetic field is removed and the restoring force in the cantilever beam pulls the electrical contacts apart.

To create a suitable switch, a number of physical design parameters must be considered. Large actuation forces are desirable for the proposed application of the microswitch. The larger the force generated, the stiffer the cantilever beam that can be employed. A stiffer beam makes for a device with faster switching time, higher gforce tolerance and greater contact breaking force. Published research [6] has shown that for common contact materials such as gold and silver, contact forces between $100 \mu \mathrm{N}$ and $1 \mathrm{mN}$ are necessary to minimize contact resistance. Large actuation forces can be used to produce large deflections allowing contact gap distances to be large. The larger the gap, the higher the device breakdown voltage [6]. Switching lifetime is also found to increase with high contact breaking force as stronger contact-to-contact welding points, which can occur during closure, can be broken.

The forces and deflections that can be generated with external magnetic fields are quite large and have been well characterized by Liu and Tai [7]. It can be shown (see figure 3) that the external magnetic field generates a large bending force on the magnetic plate which is described by equation (1). To maximize the force generated on the end of the plate, equation (1) shows that $M_{S}, W, T$ and $H$ should be maximized and that $\theta$ should be minimized. In the case of the magnetostatic switch, $H$ and $\theta$ are dictated by the target motor and the desired contact gap. In a typical DC brushless motor, $H \approx 2500$ gauss is a reasonable available magnetic field. The small contact gap, relative to the overall size of the switch, effectively eliminates the plate rotation so that $\theta \approx 0$.

To maximize $M_{S}, W$ and $T$, permalloy $\left(\mathrm{Ni}_{80} \mathrm{Fe}_{20}\right)$ is chosen to form the plate. Using this soft magnetic material eliminates the need

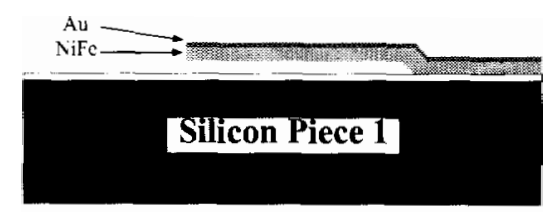

(a)

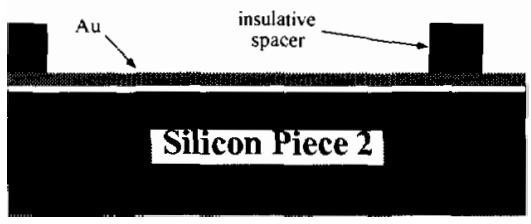

(b)

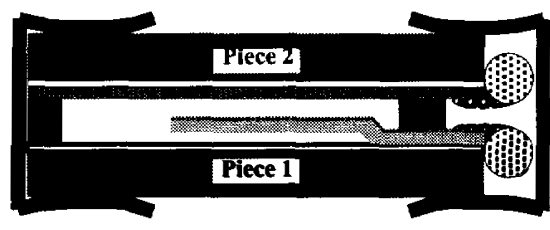

(c)

Figure 2. Switch Fabrication: (a) Create a free-standing, permalloy plate on a rigid substrate. (b) Coat an second substrate with contact material then deposit and pattern spacer. (c) Attach bond wires to pieces. Flip piece 2 over and bond with piece 1 to complete switch. to align the $M_{s}$ field of the switches with the applied external field of motor magnets. The permalloy automatically magnetizes with the proper magnetization orientation during actuation. With a high $M_{S}$ value ( $>0.8$ Tesla) and thick plating capability (tens of microns), the permalloy makes large forces possible. Based on the performance constraints for the switch, magnetic plate dimensions of $W=3 \mathrm{~mm}$ and $T=10 \mu \mathrm{m}$ were chosen to produce actuation forces in excess of $5 \mathrm{mN}$.

\section{FABRICATION}

Fabrication may be achieved with either a single substrate or dual substrate design. Because implementation as a dual substrate makes analyzing switch performance and contact wear much easier, the first generation of devices are fabricated using this approach. The first half of the switch is the permalloy plate which acts as the moving element in the switch. Its mating part is simply a substrate coated with a contact material with a spacer layer.

Processing of the first of the two pieces begins on an insulated substrate. A sacrificial spacer layer is deposited to a thickness ranging from 2 to $20 \mu \mathrm{m}$ and patterned to provide anchor holes to the substrate. Over the spacer, an electroplating seedlayer is deposited and a photoresist plating mold is laid down and patterned. Permalloy is plated to a thickness of 10 to $20 \mu \mathrm{m}$. The permalloy is then coated with contact material which, in the case of the data presented in this paper, is gold. The last step is the removal of the sacrificial spacer producing a free-standing permalloy plate anchored to the substrate.

The second piece of the dual substrate design is very simple. Contact material is laid down on a rigid substrate followed by the deposition and patterning of a permanent spacer layer ranging in thickness from 10 to $200 \mu \mathrm{m}$. This approach permits experiments with a wide range of contact materials to be conducted. As with the first substrate, this paper present data for a contact substrate of gold.

As the last step in the process, the two substrate are assembled into the final device as shown schematically in figure 2. Note that the two pieces are not permanently bonded together permitting the switch to be non-destructively disassembled. This facilitates easy access to the contact regions so that wear and failure analysis can be conducted via microscope and SEM.

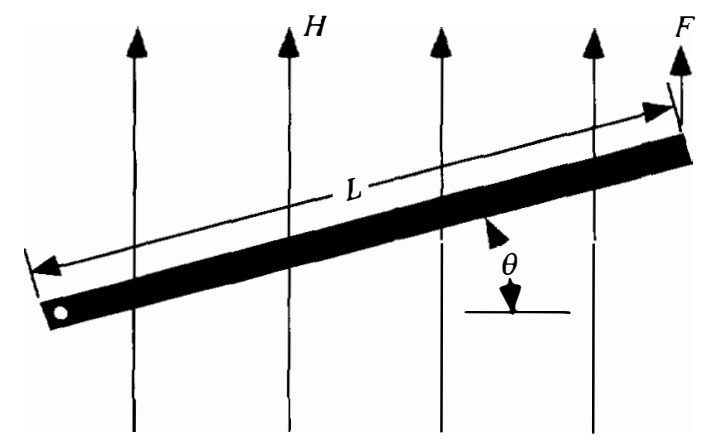

$$
\begin{gathered}
F=\text { Bending Force }=M_{S}(W T) H \cos \theta \\
T=\text { plate thickness } \quad W=\text { plate width } \\
L=\text { plate length } \quad \theta=\text { plate deflection angle } \\
H=\text { external magnetic field strength } \\
M_{S}=\text { saturation magnetization }
\end{gathered}
$$

Figure 3. Schematic showing theoretical forces generated on a plate of soft magnetic material by an externally applied magnetic field. 


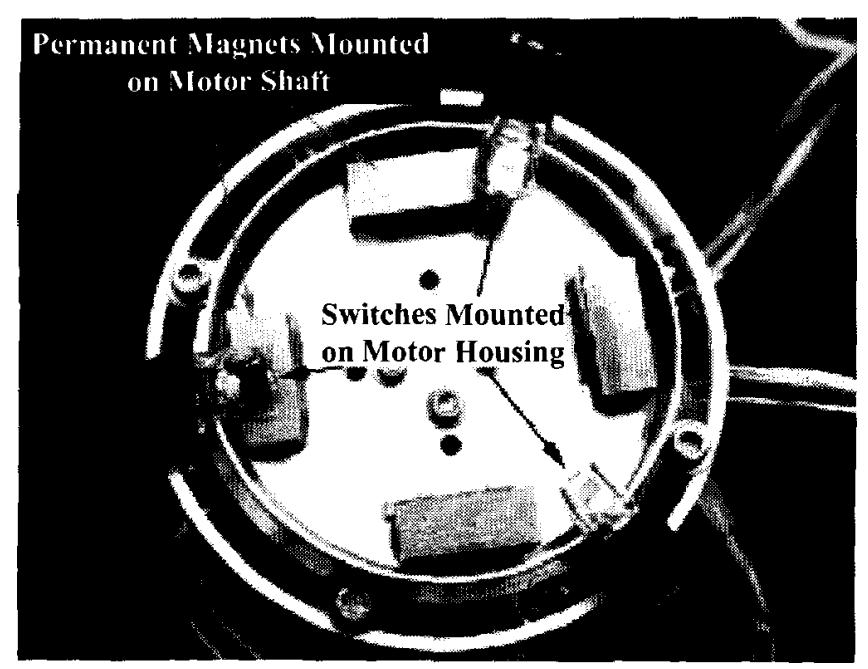

Figure 5. Photo of three switches mounted to the housing of a 3phase, DC brushless motor: Attached to the shaft of the motor are four permanent magnets mounted on a disk. Applying a single DC voltage to the motor makes it to turn without any external controlling electronics.

\section{EXPERIMENTAL SECTION}

\section{Characterization}

Static beam deflection and mechanically-simulated switch testing has been used to examine contact resistance $\left(\mathrm{R}_{\text {contact }}\right)$ vs. contact force $\left(\mathrm{F}_{\text {contact }}\right)$. Testing was performed on a setup capable of monitoring force as a function of deflection. During loading, fourpoint-resistance data were collected from the switch generating the graphs shown in figure 4. This shows that a contact force of $3-5 \mathrm{mN}$ is required to achieve an $R_{\text {contact }}$ of less than 25 milliohms and that the minimum $R_{\text {contact }}$ achievable is 1-2 milliohms. It can also be seen that the $\mathrm{R}_{\text {contact }}$ maintains slightly lower values for decreasing $\mathrm{F}_{\text {contact }}$ as compared to increasing $\mathrm{F}_{\text {contact }}$. For the beams tested and with a contact gap of $100 \mu \mathrm{m}$, the data show that approximately $1 \mathrm{mN}$ of the total bending force is required for plate deflection producing a switch with a significant break force.

Magnetic switch actuation testing has been performed on a target 3 phase, four pole DC brushless motor. The motor with three mounted switches is shown in figure 5. To simplify mounting, switches were not installed inside the motor as they will be in the final implementation. As such, the motor magnets could not be used as the source of the actuating external magnetic field. Instead, four

\section{Force vs. Deflection}

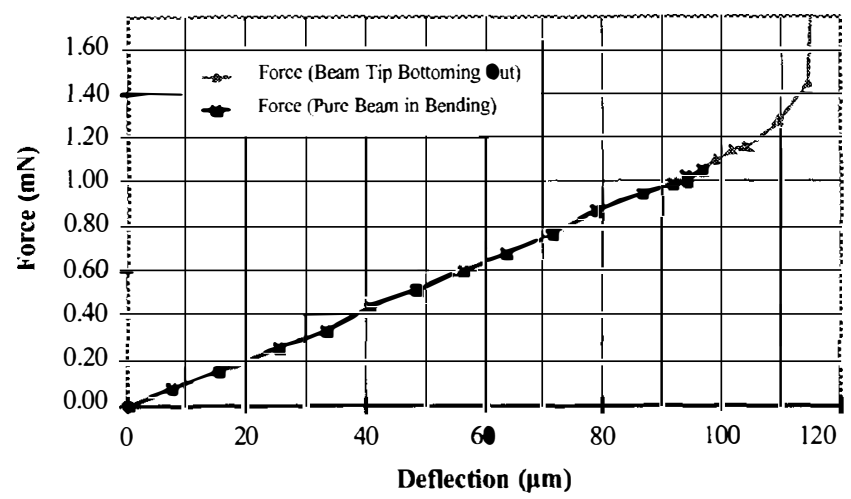

Resistance vs. Force

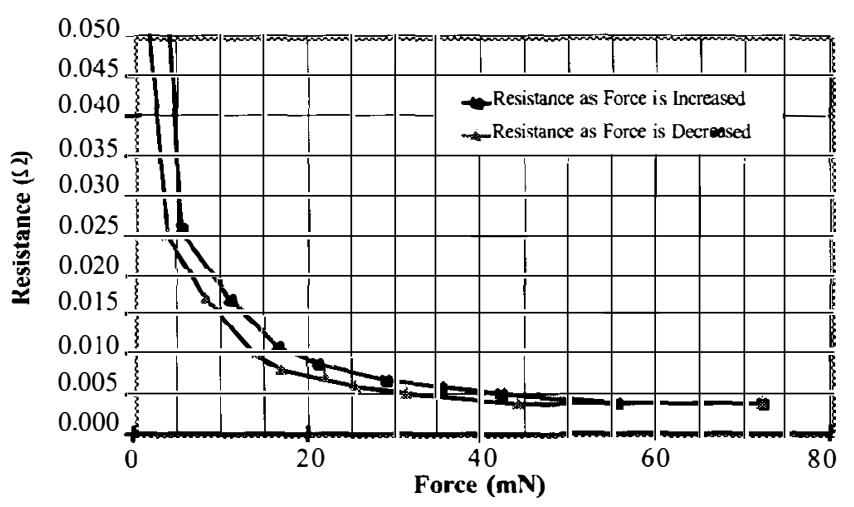

Figure 4. Graphs showing the linear beam-in-bending and contact resistance vs. contact force behavior of a MEMS magnetostatic switch.

Switch Close

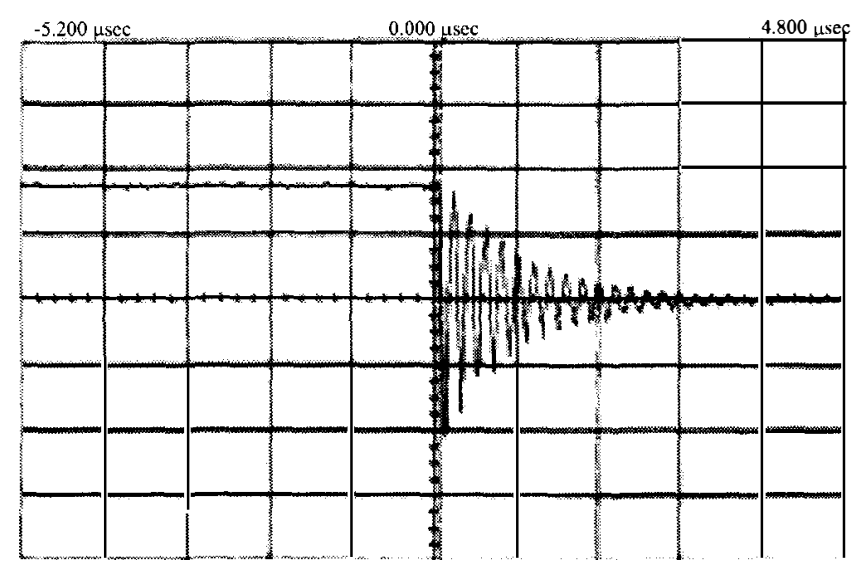

Switch Open

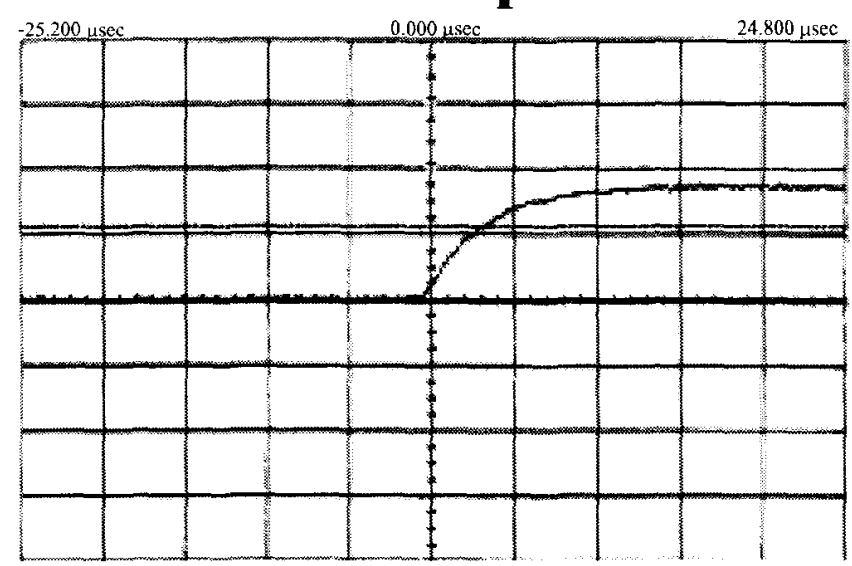

Figure 6. Oscilloscope traces of the voltage across switch as it opens and closes. The non-ideal response is due to test circuit RC parasitics. The ringing exponential decay occurs as the switch closure adds an inductance to the testing circuit. 

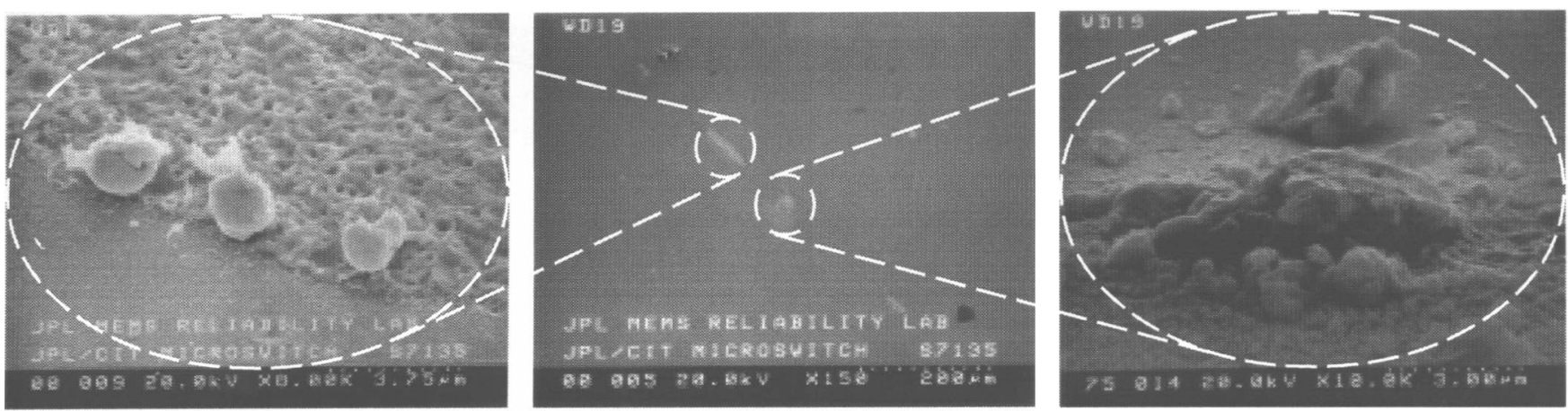

Figure 7. Center SEM shows the contact points ablated in the solid contact plate of a switch used to commutate a DC brushless motor. The contact points fall along a straight line de fined by the end of the permalloy flap (not shown). The left SEM shows damage due to melting of the contact material. The right SEM shows contact material that has been ablated from the plate and redeposited on the contact plate.

permanent magnets that generated field comparable to the motor magnets $(\approx$ 2500 gauss) were attached to an aluminum plate which was mounted to the motor's external shaft. Mounted to the motor housing, three switches are positioned at $120^{\circ}$ intervals around the motor so that they extend over the magnets. With the motor turning, fine alignment is performed to procisely position each switch to minimize torque ripple. Winding currents for this motor range from 100 to $200 \mathrm{~mA}$ at $\mathrm{DC}$ voltages ranging from 5 to 36 volts at up to 2000 RPM.

Characterization of the switching performance has been conducted using this motor setup. Switches mounted on this motor have $\mathrm{R}_{\text {contact }}<35$ milliohms when closed. Oscilloscope traces of switch opening and closure are shown in figure 6. Switch bounce on closure is not seen. The resonance on closure and exponential rise on opening are caused by circuits parasitics and are not due to the switch itself. The traces indicate that contact make and break times are less than $2 \mu \mathrm{sec}$, however this is not the switching frequency of the devices. The rise and fall of the external magnetic field seen by the switches is not a sharp transition. Instead, the field ramps gradually from zero to 2500 gauss as the motor rotation brings the permanent magnets beneath the switches. This produces "soft-switching" as the contact points on the permalloy plate "slowly" make and break eliminating contact bounce. A maximum switching speed, limited by the capability of the motor testing setup, of up to $120 \mathrm{~Hz}$ has been measured.

\section{Lifetime Testing and Contact Failure}

Extensive experimental hot-switched testing has been completed at $100 \mathrm{~Hz}$ with an on-duty-cycle of $37 \%$. The external magnetic field of $\approx 2500$ gauss translates into a switch contact force $>5 \mathrm{mN}$. Under low switching currents $(1 \mathrm{~mA})$ with a resistive load, no failure is seen after more than $5 \mathrm{x}$ $10^{8}$ cycles. Reduced lifetimes of $10^{5}-10^{6}$ cycles are seen under high current $(0.45 \mathrm{~A})$. As expected, switching of inductive loads also limits switch lifetimes. Hot switching 0.25 amps through a $4.2 \mathrm{mH}$ inductive load results in switch failure after $10^{5}-10^{6}$ cycles. Significant contact arcing is observed in this case. On the whole, the performance of the MEMS switch is comparable to commercial reed relays.

Installed on the testing motor, commutation using three magnetostatic switches has been realized. To maximize operation time, zener diodes were placed across the switches to provide arc suppression. While this does reduce electric discharge, arcing still occurs. Even so, the test motor has been successfully commutated for several hours at several hundred RPM without failure. Additionally, motor operation in vacuum, necessary for space applications, has been realized.

Study of the failure mechanism under hot cycle testing has been performed. Evidence of metal melt, splatter and redeposition from one contact onto its opposing contact is seen. The SEMs in figure 7 show the damage to two contact areas. It should be noted that as fabricated, the contact "points" were designed as the entire width of the edge of the bending plate. They are not distinct, predefined areas. As such, the melting and redeposition occurs along an intermittent line spanning the width of the plate. Failure is predominately characterized by a drastic rise in $R_{\text {contact }}$ on the order of $10^{6} \mathrm{ohms}$ although welding of the contacts resulting in permanent shorting of the switch is occasionally seen.

\section{CONCLUSIONS}

Design, characterization and successful application of a magnetostatic switch is presented. Using several of these devices, the relatively large and complex electronics packages used to commutate miniature DC brushless motors can be eliminated. This results in a substantial reduction of size, weight and complexity of the final motor system. The present design, employing gold-to-gold contacts, has produced devices capable of commutating motors at up to 36 volts and $200 \mathrm{~mA}$ for at least several hours. With proper selection of contact materials, lifetimes should be able to be significantly extended and switching of larger currents made possible.

\section{ACKNOWLEDGMENTS}

This work is supported by NASA-JPL through JPL task, number RF295. Special thanks to Michael R. Johnson for his work in designing and building the test setup and providing insight into integrating the switches with the DC brushless motor.

\section{REFERENCES}

[1] M. A. Gottschalk, "Miniature Motors Deliver Big Performance," Design News, v52, n9, p 67, May 5, 1997.

[2] C. Liu, T. Tsao, Y.C. Tai, et al., "A Micromachined Permalloy Magnetic Actuator Array for Micro Robotics Assembly Systems," Tech. Digest, 8th Int'l Conf. Solid-State Sensors and Actuators (Transducers '95/ Eurosensors IX), Stockholm, Sweden, Vol. 1, pp. 328-331, Jun. 25-29 (1995).

[3] R. A. Miller, G. W. Burr, Y. C. Tai, et al., "Electromagnetic MEMS Scanning Mirrors For Holographic Data Storage," Tech. Digest, SolidState Sensor and Actuator Workshop, Hilton Head Island, SC, pp. 183186, June 3-6 (1996).

[4] R. Miller, G. Burr, Y.C. Tai and D. Psaltis, "A Magnetically Actuated MEMS Scanning Mirror," Proc. SPIE, Miniaturized Systems With Micro-Optics and Micromachining, Vol. 2687, pp. 47-52, Jan. 1996.

[5] R. Miller, G. Burr, Y.C. Tai and D. Psaltis, "Magnetically Actuated Micromirrors For Use As Optical Deflectors," Proc ElectroChem Soc, Vol. 95-18, pp. 474-480, Oct. 1995.

[6] H. Hosaka, H Kuwano and K. Yanagisawa, "Electromagnetic Microrelays: Concepts and Fundamental Characteristics," Proc. IEEE Microelectromechanical Sys. Conf., pp. 12-17, 1993.

[7] C. Liu, T. Tsao and Y. C. Tai, "Out of Plane Permalloy Magnetic Actuators for Delta-Wing Control," Proc, 1995 IEEE Micro Electro Mech. Sys. Workshop (MEMS '95), Amsterdam, The Netherlands, pp. 7-12, Jan. 29-Feb. 2 (1995). 